\title{
Non-random X chromosome inactivation in Aicardi syndrome
}

\author{
Tanya N. Eble $\cdot$ V. Reid Sutton · Haleh Sangi-Haghpeykar $\cdot$ Xiaoling Wang • \\ Weihong Jin · Richard A. Lewis · Ping Fang • Ignatia B. Van den Veyver
}

Received: 9 October 2008 / Accepted: 19 December 2008 / Published online: 1 January 2009

(C) The Author(s) 2008. This article is published with open access at Springerlink.com

\begin{abstract}
Most females have random X-chromosome inactivation (XCI), defined as an equal likelihood for inactivation of the maternally- or paternally-derived $\mathrm{X}$ chromosome in each cell. Several X-linked disorders have been associated with a higher prevalence of non-random XCI patterns, but previous studies on XCI patterns in Aicardi syndrome were limited by small numbers and older methodologies, and have yielded conflicting results. We studied XCI patterns in DNA extracted from peripheral blood leukocytes of 35 girls with typical Aicardi syndrome (AIC) from 0.25 to 16.42 years of age, using the human androgen receptor assay. Data on 33 informative samples showed non-random XCI in 11 (33\%), defined as a >80:20\% skewed ratio of one versus the other $\mathrm{X}$ chromosome being active. In six (18\%) of these, there was a $>95: 5 \%$ extremely
\end{abstract}

T. N. Eble · V. R. Sutton · W. Jin · R. A. Lewis · P. Fang ·

I. B. Van den Veyver

Department of Molecular and Human Genetics,

Baylor College of Medicine, Houston, Texas, USA

e-mail: teble@bcm.edu

H. Sangi-Haghpeykar · X. Wang · I. B. Van den Veyver $(\square)$

Department of Obstetrics and Gynecology,

Baylor College of Medicine, 1709 Dryden,

Suite 1100, Houston, TX 77030, USA

e-mail: iveyver@bcm.edu

R. A. Lewis

Department of Ophthalmology,

Baylor College of Medicine, Houston, Texas, USA

R. A. Lewis

Department of Medicine, Baylor College of Medicine,

Houston, Texas, USA

R. A. Lewis

Department of Pediatrics, Baylor College of Medicine,

Houston, Texas, USA skewed ratio of one versus the other $\mathrm{X}$ chromosome being active. XCI patterns on maternal samples were not excessively skewed. The prevalence of non-random XCI in Aicardi syndrome is significantly different from that in the general population $(p<0.0001)$ and provides additional support for the hypothesis that Aicardi syndrome is an $\mathrm{X}$-linked disorder. We also investigated the correlation between $\mathrm{X}$-inactivation patterns and clinical severity and found that non-random XCI is associated with a high neurological composite severity score. Conversely, a statistically significant association was found between random $\mathrm{XCI}$ and the skeletal composite score. Correlations between $\mathrm{X}$-inactivation patterns and individual features were made and we found a significant association between vertebral anomalies and random XCI.

\section{Introduction}

Aicardi syndrome (AIC) is a severe sporadic neurodevelopmental disorder, originally characterized by a triad of agenesis of the corpus callosum, chorioretinal lacunae, and infantile spasms (Aicardi et al. 1965). More recent studies have identified a broader range of abnormalities with a wide variability in severity and often asymmetry (Aicardi 1999, 2005). Brain abnormalities seen in Aicardi syndrome include complete or partial agenesis of the corpus callosum, periventricular and subcortical heterotopias, polymicrogyria, microcephaly, ventriculomegaly, arachnoid cysts, cerebral asymmetry, inferior vermian hypoplasia, dysplastic and hypoplastic cerebellar hemispheres, enlarged cisterna magna, choroid plexus papillomas, and other intracranial cysts (Font et al. 1991; Hopkins et al. 2008; Menezes et al. 1994; Smith et al. 1996). Affected individuals often have severe infantile spasms that progress with age to complex 
seizures of varying types (Glasmacher et al. 2007). Electroencephalograms (EEG) are often abnormal with asynchrony between the two hemispheres, burst-suppression, and atypical hypsarrhythmia (Fariello et al. 1977; Ohtsuka et al. 1993; Palmer et al. 2006). Ophthalmological abnormalities are also variable and include unilateral or bilateral chorioretinal lacunae, optic nerve dysplasia, and microphthalmia (Aicardi 1999; Denslow and Robb 1979; Menezes et al. 1996). Other features present in subsets of affected individuals are costovertebral anomalies, facial dysmorphism, hand abnormalities, hypopigmentary skin lesions, multiple nevi, and an increased occurrence of rare vascular malformations and tumors (Sutton et al. 2005).

Aicardi syndrome has been reported nearly exclusively in females. The few known males with AIC have a 47,XXY karyotype (Hopkins et al. 1979), and reported cases of male AIC with a 46,XY karyotype have not been confirmed or may represent cases with rare mosaic mutations (Chappelow et al. 2008; Aggarwal et al. 2000; Aicardi 1980; Curatolo et al. 1980). A longstanding hypothesis is that the condition is caused by a heterozygous mutation in a gene on the $\mathrm{X}$ chromosome in females that causes lethality when present in hemizygous males (Van den Veyver 2002). Because AIC is rare and almost always sporadic, classic linkage analysis to localize the mutated gene is not possible. The lack of familial cases, aside from two reported affected sisters (Molina et al. 1989), suggests that most mutations are de novo.

The asymmetry and variability of the phenotype suggest that the putative gene mutated in AIC is subject to X-chromosome inactivation (Aicardi 2005; Van den Veyver 2002). The presumed male-lethality implies that the gene must have an important role in development and that affected females might show excess non-random XCI patterns. Until the disease-causing mutation is identified, the recognition and documentation of non-random XCI in AIC would provide additional strong support for the assertion that it is an X-linked disorder. Previous work has demonstrated that skewed $\mathrm{X}$-chromosome inactivation is common in female subjects of families with X-linked mental retardation (Plenge et al. 2002). However, the evaluation of XCI in AIC has been limited by the small numbers of subjects studied and older methodology, and different studies have generated contradictory results. One investigation, that evaluated XCI by DNA methylation analysis at the androgen receptor gene in peripheral blood leukocytes of ten females with AIC and their parents, found that patients had a random XCI pattern (Hoag et al. 1997). An isolated case report described a single girl with AIC and random XCI (Wieacker et al. 1985). In contrast, Neidich et al. observed a skewed pattern of XCI in three out of seven affected girls using methylation-sensitive restriction enzyme analysis coupled with segregation of the active $\mathrm{X}$ chromosome in somatic cell hybrids (Neidich et al. 1990). In this small series, three of the five most severely affected girls had nonrandom XCI.

To address more definitely whether individuals with AIC have excess skewing of XCI, we performed detailed clinical assessments and XCI studies in 35 peripheral blood leukocyte-derived DNA samples from girls with wellcharacterized AIC. We further investigated the relationship between the extent of XCI skewing and the severity of the phenotype in affected girls. We show that the fraction of girls with non-random XCI is higher than in the general female population.

\section{Methods}

Subjects

Individuals with AIC and their parents were enrolled under a protocol approved by the Baylor College of Medicine Institutional Review Board for Human Subjects Research. Informed consent was obtained for all study participants from the legally authorized representative. The diagnosis of AIC was confirmed in all affected patients based on phenotypic data obtained from clinical examinations, ophthalmological examinations, parental interviews, and from review of available medical records and imaging studies. The results were recorded in a secure database, as previously reported in part (Hopkins et al. 2008; Sutton et al. 2005).

Samples and DNA extraction

DNA samples from 35 females with AIC were analyzed. For 19 of these, we also sampled DNA from both parents; for ten, we only had maternal DNA samples, for one we only had a paternal DNA sample and for five we had neither maternal nor paternal DNA available. All DNA was extracted using the Puregene DNA extraction kit according to the manufacturer's protocol (Gentra Systems, Inc. Minneapolis, MN) from peripheral blood leukocytes.

$\mathrm{X}$ chromosome inactivation assay

We performed the human androgen-receptor X-inactivation assay as previously described (Allen et al. 1992; Wang et al. 2007). Briefly, $100 \mathrm{ng}$ of each female genomic DNA sample was digested either with the methylation-sensitive restriction enzyme HpaII (New England Biolabs, Inc., Ipswich, MA) or incubated with $1 \times$ enzyme buffer only. Paternal DNA samples were incubated with $1 \times$ enzyme buffer only. One nanogram of each was then amplified by PCR with primers flanking the polymorphic androgen receptor CAG repeat (Wang et al. 2007). PCR products 
were separated by capillary electrophoresis on an $\mathrm{ABI}$ Prism 3100 Genetic Analyzer and analyzed with GeneMapper V3.5 software (Applied Biosystems, Foster City, CA). The percent $\mathrm{X}$-chromosome inactivation was calculated with the following formula: percent inactivation $=$ allele A peak height/(allele A peak height + allele B peak height $\times 100$. We used a cutoff of $>80: 20 \%$ for skewed XCI and $>95: 5 \%$ for extremely skewed XCI (Allen et al. 1992; Naumova et al. 1996).

\section{Phenotype-XCI correlation}

Phenotypic features for each of the individuals with AIC, gathered from the ophthalmological examination $(n=32)$, neuroimaging studies $(n=24)$, parental interview $(n=27)$, and review of medical records $(n=25)$ were given a weighted score (Table 1). Composite severity scores (CSS) for overall severity, neurological, skeletal, and ophthalmological findings were also calculated. Ophthalmological CSS features include microphthalmia, lacunae, and optic nerve abnormalities, neurological CSS features include microgyria, ACC, heterotopias and cysts, skeletal CSS features include absent or abnormal ribs and vertebral anomalies and all features were included for the overall CSS. The severity of individual phenotypes and CSS were compared between girls with random XCI and girls with non-random XCI. The correlation between overall CSS and XCI patterns was calculated in two different ways: the first including only patients with values for each one of the phenotypes examined, and the second including all patients with any phenotypic data, even if there were missing values for some of the examined phenotypes.

\section{Statistical analysis}

Statistical comparison of the groups of interest was made via $X^{2}$ or Fisher exact test for grouped data, and Wilcoxon rank test for ordinal data. Spearman correlation coefficients were generated to assess the degree of relationship between subject's age and percent XCI. A $p$ value $\leq 0.05$ was considered statistically significant. All analyses were performed with the SAS System statistical software (SAS, Cary, NC).

\section{Results}

The ages of the affected girls at the time the DNA samples were obtained ranged from 0.25 to 16.42 years, with a mean age of 5.12 years and a median age of 4.84 years. Skewed $(>80: 20 \%)$ XCI was seen in $11(33 \%)$ of the girls, of whom six (18\%) showed extreme skewing ( $>95: 5 \%)$ (Fig. 1a). We compared the prevalence of XCI skewing in
Table 1 Phenotypic scoring system

\begin{tabular}{|c|c|c|c|}
\hline Phenotypic Feature & Finding & Score & $\mathrm{N}(\%)$ \\
\hline \multirow{3}{*}{$\begin{array}{l}\text { Agenesis of the } \\
\text { Corpus Callosum } \\
(N=33)\end{array}$} & No & 0 & $0(0)$ \\
\hline & Partial & 1 & $11(33.3)$ \\
\hline & Complete & 2 & $22(66.7)$ \\
\hline \multirow{2}{*}{$\begin{array}{l}\text { Infantile Spasms } \\
\qquad(N=31)\end{array}$} & No & 0 & $2(6.5)$ \\
\hline & Yes & 1 & $29(93.5)$ \\
\hline \multirow[t]{2}{*}{$\operatorname{Microgyria}^{\mathrm{a}}(N=21)$} & No & 0 & $2(9.5)$ \\
\hline & Yes & 1 & $19(90.5)$ \\
\hline \multirow[t]{2}{*}{ Heterotopias $^{\mathrm{a}}(N=22)$} & No & 0 & $3(13.6)$ \\
\hline & Yes & 1 & $19(86.4)$ \\
\hline \multirow{2}{*}{$\begin{array}{l}\text { Intracranial Cysts }^{\mathrm{a}} \\
\quad(N=26)\end{array}$} & No & 0 & $4(15.4)$ \\
\hline & Yes & 1 & $22(84.6)$ \\
\hline \multirow{4}{*}{$\begin{array}{l}\text { Retinal Lacunae }^{\mathrm{b}} \\
\qquad(N=31)\end{array}$} & No & 0 & $2(6.5)$ \\
\hline & Unable to Assess & 1 & $1(3.2)$ \\
\hline & Unilateral & 2 & $3(9.7)$ \\
\hline & Bilateral & 3 & $25(80.6)$ \\
\hline \multirow{3}{*}{$\begin{array}{l}\text { Microphthalmia }{ }^{\mathrm{b}} \\
\quad(N=31)\end{array}$} & No & 0 & $21(67.8)$ \\
\hline & Unilateral & 2 & $9(29)$ \\
\hline & Bilateral & 3 & $1(3.2)$ \\
\hline \multirow{4}{*}{$\begin{array}{l}\text { Optic Nerve } \\
\text { Abnormality } \\
(N=31)\end{array}$} & No & 0 & $2(6.5)$ \\
\hline & Unable to Assess & 1 & $2(6.5)$ \\
\hline & Unilateral & 2 & $10(32.2)$ \\
\hline & Bilateral & 3 & $17(54.8)$ \\
\hline \multirow{2}{*}{$\begin{array}{l}\text { Facial asymmetry } \\
\qquad(N=26)\end{array}$} & No & 0 & $23(88.5)$ \\
\hline & Yes & 1 & $3(11.5)$ \\
\hline \multirow{2}{*}{$\begin{array}{l}\text { Absent or Abnormal } \\
\operatorname{Ribs}^{c}(N=25)\end{array}$} & No & 0 & $18(72)$ \\
\hline & Yes & 1 & $7(28)$ \\
\hline \multirow{2}{*}{$\begin{array}{l}\text { Vertebral } \\
\qquad \begin{array}{l}\text { Abnormalities } \\
\text { c }\end{array} \\
(N=21)\end{array}$} & No & 0 & $11(52.3)$ \\
\hline & Yes & 1 & $10(47.6)$ \\
\hline
\end{tabular}

Phenotypic features of girls with AIC were given a weighted score relative to their severity or to their unilateral or bilateral presence. All values were added to calculate an overall composite severity score (CSS) for each individual

${ }^{\text {a }}$ Values for selected features were added to calculate a neurological CSS for each individual

b Values for selected features were added to calculate a ophthalmological CSS for each individual

c Values for selected features were added to calculate a skeletal CSS for each individual

our study population to that in the general population as reported in Amos-Landgraf et al. 2006 who used the same $>80: 20 \%$ and $>95: 5 \%$ cut-offs to define XCI skewing and the same methodology as used in our study. We found that the prevalence of XCI skewing $(p<0.0001)$ and of extreme XCI skewing $(p<0.0001)$ were both significantly increased over that reported for the general population.

Only three (9\%) of studied mothers had a non-random XCI pattern. Interestingly, when XCI patterns in motherdaughter pairs were compared, we found two pairs for 

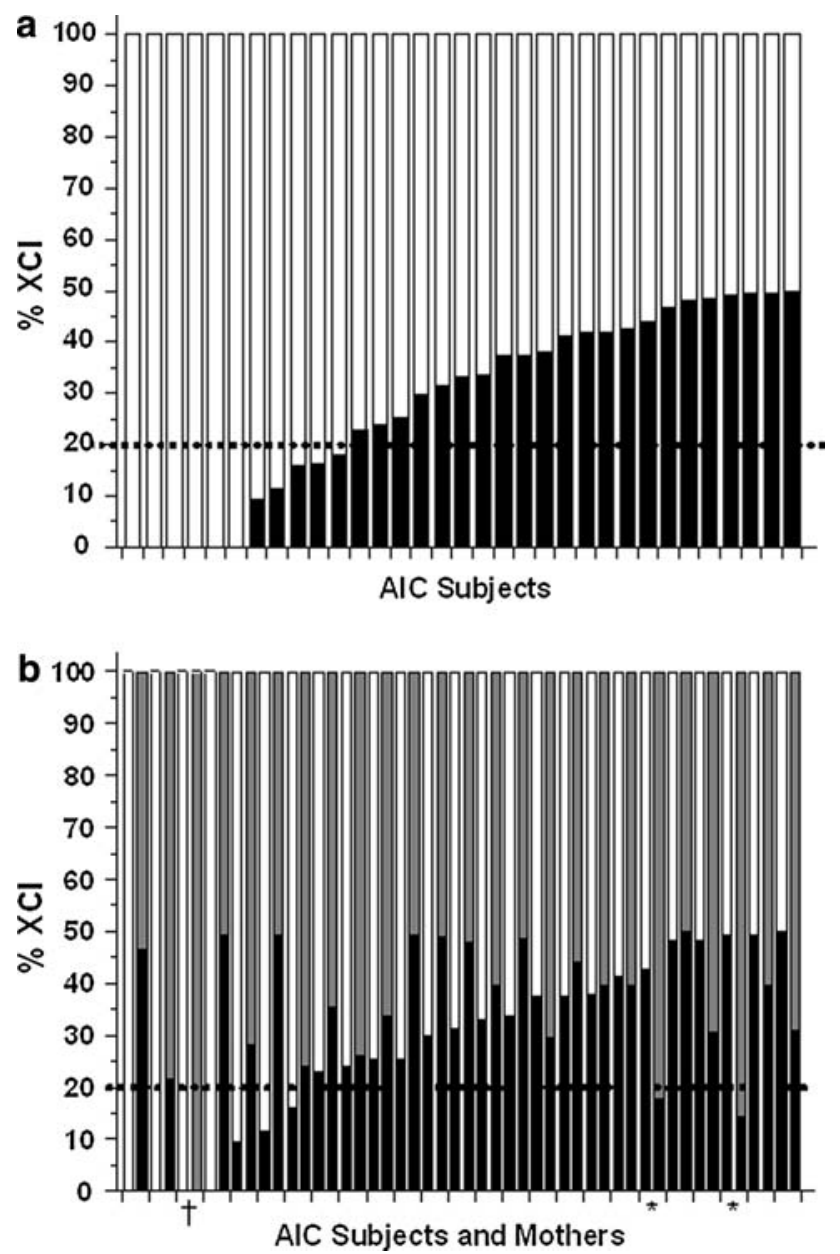

Fig. 1 Patterns of $X$ chromosome inactivation in girls with Aicardi syndrome and their mothers. The \% XCI is shown for each AIC girl (a) and for mother-girl pairs (b). a includes only 33 bars because two of the 35 girls tested had non-informative results. Twenty-five mothergirl pairs for which informative results were available for both mother and daughter are included in $\mathbf{b}$. The active $\%$ of one versus the other $\mathrm{X}$-chromosome is represented as black-and-white vertical bars for AIC girls and as black-and-grey vertical bars for mothers. The areas on the graphs below the hatched line delineate the $80: 20 \%$ cut off for skewing. Asterisks indicate pairs in which the mother has skewed XCI while the daughter has random XCI. Dagger indicates a pair in which both mother and daughter have extremely skewed XCI

whom the mother had skewed XCI while the daughter has random XCI. In addition, there was extremely skewed XCI in one mother-daughter pair (Fig. 1b). Our current data do not reflect a significant correlation between age and percentage of XCI in the girls with Aicardi syndrome $(R=-0.12 ; p=0.25)$ (data not shown). This may be related to sample size, the young age and the limited age range of our study population ( $0.25-16.42$ years).

For nine girls with skewed or extremely skewed XCI for when we could determine the parental origin of the preferentially inactivated $\mathrm{X}$-chromosome, we found that the paternal $\mathrm{X}$ and the maternal $\mathrm{X}$ were equally likely to be preferentially inactivated with the paternal $\mathrm{X}$ inactivated in
Table 2 Correlation of XCI with severity of the AIC phenotype

\begin{tabular}{llll}
\hline $\begin{array}{l}\text { Composite } \\
\text { severity score }\end{array}$ & $\begin{array}{l}\text { Mean CSS } \\
\text { random } \\
\text { XCI }(n=23)\end{array}$ & $\begin{array}{l}\text { Mean CSS } \\
\text { non-random } \\
\text { XCI }(n=11)\end{array}$ & $\begin{array}{l}\text { Two-sided } \\
P \text { value } \\
\text { random versus } \\
\text { non-random }\end{array}$ \\
\hline $\begin{array}{l}\text { Neurological } \\
\quad(N=17)\end{array}$ & 4.18 & 5.0 & 0.049 \\
$\begin{array}{l}\text { Ophthalmological } \\
(N=26)\end{array}$ & 3.77 & 3.67 & 0.96 \\
$\begin{array}{c}\text { Skeletal }(N=19) \\
\text { Overall }{ }^{\mathrm{a}}(N=7)\end{array}$ & 1.0 & 0.3 & 0.05 \\
$\begin{array}{c}\text { Overall CSS } \\
(N=31)\end{array}$ & 8.5 & 9.5 & 0.20 \\
$\quad$ & 8.2 & 0.80
\end{tabular}

Mean scores for each CSS were calculated by averaging the number of scored features observed in each participant. $P$ values are from the Wilcoxon rank test. Two types of correlations with overall CSS were preformed

${ }^{a}$ Includes only patients with values for each one of the phenotypes examined

b Includes all patients with any available phenotype data, even if there were missing values

five (55\%) versus the maternal $\mathrm{X}$ inactivated in four (45\%) girls.

To evaluate whether skewing of XCI was associated with severity of the phenotype, we scored key distinctive features of AIC (Table 1) for severity and derived an overall, neurological, skeletal and ophthalmological composite severity scores (CSS) (Table 2). We then compared the severity of individual features and the composite severity scores between the groups with random and non-random $\mathrm{XCI}$ patterns. For the individual features we found a statistically significant association between the presence of vertebral abnormalities and a random pattern of XCI $(p=0.05)$. We also found that girls with non-random XCI had a higher mean neurological composite severity score compared to girls with random XCI $(p=0.049)$ but a lower mean skeletal composite severity score compared to girls with random XCI $(p=0.05)$. There was no association between the degree of skewing and overall or ophthalmological severity scores, but this may reflect the essential role of the ophthalmological features in the diagnosis of AIC. The clinical significance of these correlations is currently unclear.

\section{Discussion}

It is thought that Aicardi syndrome is caused by the mutation of a gene or genes on the X-chromosome that are subject to XCI because it is sporadic and occurs exclusively in females or rare males with two $\mathrm{X}$ chromosomes $(47, \mathrm{XXY}$ karyotype). Studies of XCI in AIC conducted in the past 
did not provide a definitive conclusion about X-chromosome inactivation status in AIC (Neidich et al. 1990; Hoag et al. 1997; Wieacker et al. 1985). Our study demonstrates that skewing of XCI is present in a much higher number of affected girls than what would be expected, even though not all subjects have non-random patterns of XCI.

Dosage compensation for genes on the X-chromosome between females having two $\mathrm{X}$-chromosomes and males having one $\mathrm{X}$-chromosome is achieved through the mechanism of XCI (Migeon 1994). In females, most genes on one of the two X-chromosomes in each somatic cell are inactivated during early embryogenesis (Bittel et al. 2008). When somatic cells divide, the choice of which $\mathrm{X}$ is inactivated is passed on to subsequent daughter cells and the result should be an approximately equal distribution of cells with maternally and paternally inherited X-chromosomes inactive. The fraction of cells that inactivate either the maternally or paternally inherited X-chromosome follows a normal distribution in the general population. When DNA from peripheral blood cells is analyzed, extreme skewing ( $>95: 5 \%$ ) occurs in $\sim 1 \%$ of females (Bittel et al. 2008; Amos-Landgraf et al. 2006; Hatakeyama et al. 2004; Sharp et al. 2000) while overall skewing ( $>80: 20 \%$ ) occurs in $\sim 8 \%$ of females (AmosLandgraf et al. 2006). In contrast, our study showed that $33 \%$ of girls with Aicardi syndrome had skewed XCI and $18 \%$ had extremely skewed XCI. Although our study was done on a different set of DNA samples than prior reports of XCI patterns in the general population, the method and tissues used to evaluate XCI was similar. Hence, we conclude that the prevalence of extremely skewed XCI is higher than in the general population. This supports the prevailing hypothesis that $\mathrm{AIC}$ is due to a mutation in a gene on the $\mathrm{X}$-chromosome that is subject to XCI. Similarly, an increased prevalence of skewing is seen incontinentia pigmenti, a condition that is X-linked and lethal in males (Wieacker et al. 1985; Parrish et al. 1996). Selection against cells with an active mutationbearing chromosome has also been observed in female carriers of certain X-linked immunodeficiencies, such as X-linked agammaglobulinemia and X-linked severe combined immunodeficiency (Puck and Willard 1998).

There are various plausible explanations for the fact that not all individuals with Aicardi syndrome have extremely skewed or skewed XCI. Some may have milder mutations in the putative gene. Another explanation could be the presence of postzygotic mosaic mutations, as has been recently suggested to explain random XCI in another X-linked dominant disorder, Goltz syndrome (Grzeschik et al. 2007). Although confirmation will need to await discovery of the causative gene, it could explain why $67 \%$ of girls with AIC have random XCI, and might account for the wide range of phenotypes seen in affected girls. Another explanation for the observed random XCI in $67 \%$ of subjects could be the effect of age on patterns of XCI in rapidly renewing cells, such as peripheral blood leukocytes. This is observed in WiscottAldrich syndrome, where there is a post-inactivation disadvantage to cells that have the mutation-bearing X-chromosome active in women who carry heterozygous WAS mutations (Puck and Willard 1998). Although we may have underestimated the frequency of skewing in some of the girls with AIC for this reason, it is less likely because we are studying a very young population (mean age 5.12 years). Typically an increased frequency of skewed XCI is seen in the general population in women 60 years of age and older (Sharp et al. 2000).

Girls with skewed XCI were found to have significantly more severe neurological involvement than girls with random XCI. Conversely, girls with random XCI were found to be significantly more likely to have vertebral anomalies and a greater overall skeletal involvement. The clinical relevance of these observations will remain unclear until the disease-causing gene is identified.

The possibility of a mutation on an autosome with sexlimited expression rather than an X-linked mutation can theoretically not be completely eliminated as a possible cause of AIC, but the departure from the non-random XCI in our larger cohort of individuals with AIC provides additional strong evidence that it is due to a mutation in an $\mathrm{X}$-linked gene that is subject to XCI and reinforces the approach to focus the search for an appropriate candidate gene to the $\mathrm{X}$ chromosome. Because our study was performed on DNA from peripheral blood leukocytes, our results may further suggest that the putative AIC gene has a function in peripheral blood leukocytes or bone marrow cells, even though the phenotype is primarily neurological and ocular. Our combined findings will aid prioritization of candidate genes for ongoing mutation analysis studies.

Acknowledgments We wish to thank the subjects and their families for their enthusiastic participation in this research. This study was supported in part by the Aicardi Syndrome Foundation and by grants HD051805 (to IBV) and HD024064 (Baylor College of Medicine Mental Retardation and Developmental Disabilities Research Center) from the Eunice Kennedy Shriver National Institute of Child Health and Human Development. The content is solely the responsibility of the authors and does not necessarily represent the official views of the National Institute of Child Health And Human Development or the National Institutes of Health. RAL is a Senior Scientific Investigator of Research to Prevent Blindness, New York, New York.

Open Access This article is distributed under the terms of the Creative Commons Attribution Noncommercial License which permits any noncommercial use, distribution, and reproduction in any medium, provided the original author(s) and source are credited.

\section{References}

Aggarwal KC, Aggarwal A, Prasad MS, Salhan RN, Upadhaya A (2000) Aicardi's syndrome in a male child: an unusual presentation. Indian Pediatr 37:542-545 
Aicardi J (1980) Aicardi syndrome in male infant editorial correspondence. J Pediatr 97:1040-1041

Aicardi J (1999) Aicardi Syndrome: old and new findings. Int Pediatr $14: 5-8$

Aicardi J (2005) Aicardi syndrome. Brain Dev 27:164-171

Aicardi J, Levebre A, Lerique-Koechlin A (1965) A new syndrome: spasms in flexion, callosal agenesis, ocular abnormalities. Electroencephalogr Clin Neurophysiol 19:609-610

Allen RC, Zoghbi HY, Moseley AB, Rosenblatt HM, Belmont JW (1992) Methylation of HpaII and HhaI sites near the polymorphic CAG repeat in the human androgen-receptor gene correlates with X chromosome inactivation. Am J Hum Genet 51:1229-1239

Amos-Landgraf JM, Cottle A, Plenge RM, Friez M, Schwartz CE, Longshore J, Willard HF (2006) X chromosome-inactivation patterns of 1, 005 phenotypically unaffected females. Am J Hum Genet 79:493-499

Bittel DC, Theodoro MF, Kibiryeva N, Fischer W, Talebizadeh Z, Butler MG (2008) Comparison of X chromosome inactivation patterns in multiple tissues from human females. J Med Genet 45:309-313

Chappelow AV, Reid J, Parikh S, Traboulsi EI (2008) Aicardi syndrome in a genotypic male. Ophthalmic Genet 29:181-183

Curatolo P, Libutti G, Dallapiccola B (1980) Aicardi syndrome in a male infant. J Pediatr 96:286-287

Denslow GT, Robb RM (1979) Aicardi's syndrome: a report of four cases and review of the literature. J Pediatr Ophthalmol Strabismus 16:10-15

Fariello RG, Chun RW, Doro JM, Buncic JR, Prichard JS (1977) EEG recognition of Aicardi's syndrome. Arch Neurol 34:563-566

Font RL, Marines HM, Cartwright J, Bauserman SC (1991) Aicardi syndrome. A clinicopathologic case report including electron microscopic observations. Ophthalmology 98:1727-1731

Glasmacher MA, Sutton VR, Hopkins B, Eble T, Lewis RA, Parsons D, Van den Veyver IB (2007) Phenotype and management of Aicardi syndrome: new findings from a survey of 69 children. J Child Neurol 22:176-184

Grzeschik KH, Bornholdt D, Oeffner F, Konig A, n Boente M, Enders H, Fritz B, Hertl M, Grasshoff U, Hofling K, Oji V, Paradisi M, Schuchardt C, Szalai Z, Tadini G, Traupe H, Happle R (2007) Deficiency of $P O R C N$, a regulator of Wnt signaling, is associated with focal dermal hypoplasia. Nat Genet 39:833-835

Hatakeyama C, Anderson CL, Beever CL, Penaherrera MS, Brown CJ, Robinson WP (2004) The dynamics of X-inactivation skewing as women age. Clin Genet 66:327-332

Hoag HM, Taylor SA, Duncan AM, Khalifa MM (1997) Evidence that skewed $\mathrm{X}$ inactivation is not needed for the phenotypic expression of Aicardi syndrome. Hum Genet 100:459-464

Hopkins B, Sutton VR, Lewis RA, Van den Veyver IB, Clark GD (2008) Neuroimaging aspects of Aicardi syndrome. Am J Med Genet 146A:2871-2878

Hopkins IJ, Humphrey IK, Keith CG, Susman M, Webb GC, Turner EK (1979) The Aicardi syndrome in a 47, XXY male. Aust Paediatr J 15:278-280
Menezes AV, Lewis TL, Buncic JR (1996) Role of ccular involvement in the prediction of visual development and clinical prognosis in Aicardi syndrome. Br J Ophthalmol 80:805-811

Menezes AV, MacGregor DL, Buncic JR (1994) Aicardi syndrome: natural history and possible predictors of severity. Pediatr Neurol 11:313-318

Migeon BR (1994) X-chromosome inactivation: molecular mechanisms and genetic consequences. Trends Genet 10:230-235

Molina JA, Mateos F, Merino M, Epifanio JL, Gorrono M (1989) Aicardi syndrome in two sisters. Pediatr 115:282-283

Naumova AK, Plenge RM, Bird LM, Leppert M, Morgan K, Willard HF, Sapienza C (1996) Heritability of X chromosome-inactivation phenotype in a large family. Am J Hum Genet 58:1111-1119

Neidich JA, Nussbaum RL, Packer RJ, Emanuel BS, Puck JM (1990) Heterogeneity of clinical severity and molecular lesions in Aicardi syndrome. Pediatr 116:911-917

Ohtsuka Y, Oka E, Terasaki T, Ohtahara S (1993) Aicardi syndrome: a longitudinal clinical and electroencephalographic study. Epilepsia 34:627-634

Palmer L, Zetterlund B, Hard AL, Steneryd K, Kyllerman M (2006) Aicardi syndrome: presentation at onset in Swedish children born in 1975-2002. Neuropediatrics 37:154-158

Parrish JE, Scheuerle AE, Lewis RA, Levy ML, Nelson DL (1996) Selection against mutant alleles in blood leukocytes is a consistent feature in Incontinentia Pigmenti type 2. Hum Mol Genet 5:1777-1783

Plenge RM, Stevenson RA, Lubs HA, Schwartz CE, Willard HF (2002) Skewed X-chromosome inactivation is a common feature of X-linked mental retardation disorders. Am J Hum Genet $71: 168-173$

Puck JM, Willard HF (1998) X inactivation in females with X-linked disease. N Engl J Med 338:325-328

Sharp A, Robinson D, Jacobs P (2000) Age- and tissue-specific variation of $\mathrm{X}$ chromosome inactivation rations in normal women. Hum Genet 107:343-349

Smith CD, Ryan SJ, Hoover SL, Baumann RJ (1996) Magnetic resonance imagining of the brain in Aicardi's syndrome. Report of 20 patients. Neuroimaging 6:214-221

Sutton VR, Hopkins BJ, Eble TN, Gambhir N, Lewis RA, Van den Veyver IB (2005) Facial and physical features of Aicardi Syndrome: infants to teenagers. Am J Med Genet 138A:254-258

Van den Veyver I (2002) Microphthalmia with linear skin defects (MLS), Aicardi, and Goltz syndrome: are they related X-linked dominant male-lethal disorders? Cytogenet Genome Res 99:289296

Wang X, Sutton VR, Peraza-Llanes OJ, Yu Z, Rosetta R, Kou YC, Eble TN, Patel A, Thaller C, Fang P, Van den Veyver IB (2007) Mutations in X-linked PORCN, a putative regulator of Wnt signaling, cause focal dermal hypoplasia. Nat Genet 39:836-838

Wieacker P, Zimmer J, Ropers H (1985) X inactivation patterns in two syndromes with probable X-linked dominant, male lethal inheritance. Clinic Genet 28:238-242 